\title{
Interlayer adaptation-induced explosive synchronization in multiplex networks
}

\author{
Anil Kumar $\odot$, Sarika Jalan, ${ }^{*}$ and Ajay Deep Kachhvah $\odot$ \\ Complex Systems Lab, Discipline of Physics, Indian Institute of Technology Indore, Khandwa Road, Simrol, Indore 453552, India
}

(Received 22 October 2019; accepted 26 March 2020; published 1 June 2020)

\begin{abstract}
It is known that intralayer adaptive coupling among connected oscillators instigates explosive synchronization (ES) in multilayer networks. Taking an altogether different cue in the present work, we consider interlayer adaptive coupling in a two-layer multiplex network of phase oscillators and show that the scheme gives rise to ES with an associated hysteresis. The hysteresis is shaped by the interlayer coupling strength and the frequency mismatch between the mirror nodes. We provide a rigorous mean-field analytical treatment and show that the analytical predictions are in fair agreement with the numerical assessments. Moreover, the analytical predictions provide extensive insight into how adaptive interlayer coupling suppresses the formation of a giant cluster, eventually giving birth to ES. The study will help in spotlighting the role of interlayer adaptation in the emergence of ES in real-world systems represented by multilayer architecture. In particular, it is relevant to those systems which have limitations towards changes in intralayer coupling strength.
\end{abstract}

DOI: 10.1103/PhysRevResearch.2.023259

\section{INTRODUCTION}

Recently, an irreversible synchronization process, called explosive synchronization (ES), in which a group of incoherent dynamical units are abruptly set in collective coherent motion, has drawn much attention of researchers [1,2]. The abnormal hypersensitivity of ES can be perilous in many physical and biological circumstances such as abrupt cascading failure of the power grid [3], breakdown of the internet due to intermittent congestion [4], abrupt attack of epileptic seizures in the human brain [5], and abrupt episodes of chronic pain in the fibromyalgia human brain [6], to name a few. An anesthetic-induced transition to unconsciousness $[7,8]$ and bistability in a Cdc2-cyclin B oscillator [9] are other instances of an abrupt transition.

The occurrence of ES has also been demonstrated experimentally $[10,11]$. The origin of ES is shown to be rooted in inertia [12] and a microscopic correlation between frequency and degree or coupling strength of the networked phase oscillators [13,14]. Recently, Zhang et al. [15] showed that a fraction of adaptively coupled phase oscillators give birth to ES. Danziger et al. [16] further showed that explosive synchronization can coexist with classical synchronization. Adaptation is an inherent feature in the construction of many complex systems; for instance, adaptation in neural networks explains the learning or memory process $[17,18]$.

\footnotetext{
*Corresponding author: sarikajalan9@gmail.com

Published by the American Physical Society under the terms of the Creative Commons Attribution 4.0 International license. Further distribution of this work must maintain attribution to the author(s) and the published article's title, journal citation, and DOI.
}

In many complex systems, the same set of nodes may have different types of connections among them, having each type influencing the functionality of other types. Hence, an isolated network is an unfit candidate to model such systems. Such systems can be precisely framed by a multiplex network, where different layers denoting different dynamical processes are interconnected by the same set of nodes [19-28], for instance, social networks, neuronal networks, and transport systems in which individuals, neurons, and cities have different types of connections among them, each type forming a different layer [29]. The multiplex framework has been remarkably successful in providing insight into the dynamical behavior of various processes such as percolation [30], epidemic spreading $[31,32]$, opinion formation in social networks [33], and cluster synchronization [34].

Recently, the studies concerning the origin of ES have been extended to multilayer networks by employing different methodologies, for instance, adaptive coupling [15], intertwined coupling [35], inertia [36], delayed coupling [37], inhibitory coupling [38], and frequency mismatch [39]. A few recent studies on multilayer networks have adopted the adaptive coupling dynamics proposed by Zhang et al. $[40,41]$ at the heart of their models. It has been reported that the occurrence of ES in a multilayer network is exhibited by a fraction of nodes which are adaptively coupled via local order parameters within the multiplexed layers, having virtual interlayer links [15]. In the present work, taking an altogether different cue, we propose an approach in which interlayer links of a multiplex network are adaptively coupled through global order parameters of the interacting layers. The model exhibit ES with an associated hysteresis. The phenomenon is sensitive to frequency mismatch between the interconnected nodes which along with interlayer coupling strength determines the size of emergent hysteresis. We corroborate our findings by providing rigorous mean-field analysis and show a good match between the analytical prediction of the order parameter and its numerical evaluation. 


\section{MODEL AND TECHNIQUE}

We consider a multiplex network consisting of two layers, each one having $N$ nodes represented by Kuramoto oscillators. Each node in a layer is adaptively linked with its mirror node in another layer through the interlayer coupling strength, which is a function of phase coherence among nodes of the interacting layers. The time evolution of phases of the nodes in the multiplexed layers $a$ and $b$ is ruled by [42]

$$
\begin{aligned}
& \dot{\theta_{i}^{a}}=\omega_{i}^{a}+\frac{\sigma^{a}}{N} \sum_{j=1}^{N} A_{i j}^{a} \sin \left(\theta_{j}^{a}-\theta_{i}^{a}\right)+\lambda r^{a} r^{b} \sin \left(\theta_{i}^{b}-\theta_{i}^{a}\right), \\
& \dot{\theta_{i}^{b}}=\omega_{i}^{b}+\frac{\sigma^{b}}{N} \sum_{j=1}^{N} A_{i j}^{b} \sin \left(\theta_{j}^{b}-\theta_{i}^{b}\right)+\lambda r^{a} r^{b} \sin \left(\theta_{i}^{a}-\theta_{i}^{b}\right),
\end{aligned}
$$

where $i=1, \ldots, N ; \theta_{i}^{a(b)}$ and $\omega_{i}^{a(b)}$ represent the phase and natural frequency of the $i$ th node in layer $a(b)$, respectively; $\sigma^{a(b)}$ denotes the intralayer coupling strength among the nodes of layer $a(b)$; and we set $\sigma^{a}=\sigma^{b}=\sigma$. The connectivity of the nodes in the multiplexed layers is encoded into a set of adjacency matrices $\mathbf{A}=\left\{A^{a}, A^{b}\right\}$, where $A_{i j}^{a(b)}=1$ if nodes $i^{a(b)}$ and $j^{a(b)}$ are connected and $A_{i j}^{a(b)}=0$ otherwise. $\lambda$ represents the static interlayer coupling while $\lambda r^{a} r^{b}$ represents adaptive interlayer coupling. Here global order parameter $r^{a(b)}$ is a measure of phase coherence among the nodes of layer $a(b)$. It is given by

$$
r^{a(b)} e^{i \psi^{a(b)}}=\frac{1}{N} \sum_{j=1}^{N} e^{i \theta_{j}^{a(b)}},
$$

where $0 \leqslant r^{a(b)} \leqslant 1$. The minimum value $r^{a(b)}=0$ corresponds to a uniform distribution of oscillators over a unit circle, whereas the maximum value $r^{a(b)}=1$ corresponds to exact phase synchronization. Equations (1) are solved numerically using the Runge-Kutta fourth-order method with a time step $d t=0.01$. To determine phase coherence among the nodes of layer $a(b)$, the time average of $r^{a(b)}$ is taken for $10^{5}$ steps after neglecting the initial $10^{5}$ steps. The initial phases and natural frequencies of the oscillators in layers $a$ and $b$ are drawn from a uniform random distribution in the ranges $-\pi \leqslant \theta_{i}^{a(b)} \leqslant \pi$ and $-\gamma \leqslant \omega_{i}^{a(b)} \leqslant \gamma$, respectively. In this work we take $\gamma=0.5$.

\section{RESULTS}

\section{A. Adaptive interlayer coupling leads to ES}

To investigate the effect of adaptive coupling between all pairs of mirror nodes, we consider a multiplex network of two globally coupled networks, unless stated otherwise. Furthermore, oscillators in the two layers have identical natural frequency distributions, but in general $\omega_{i}^{a} \neq \omega_{i}^{b}$. In forward and backward continuation of coupling, output phases at coupling $\sigma$ are used as initial phases at couplings $\sigma+d \sigma$ and $\sigma-d \sigma$, respectively, where $d \sigma$ represents a small change in the coupling strength. Figure 1 depicts the behavior of the $r^{a}-\sigma$ profile for the considered multiplex network. In
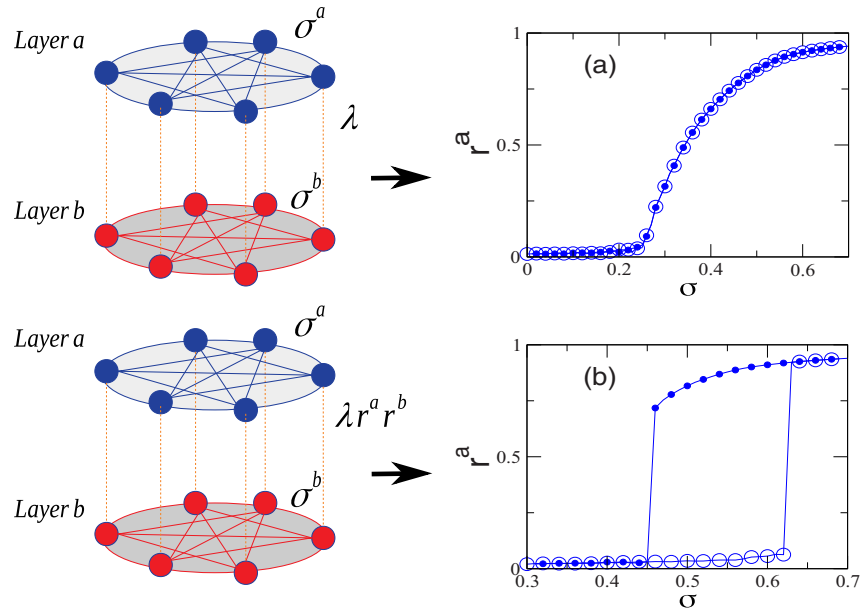

FIG. 1. (a) Static interlayer coupling $\lambda$ leads to a continuous phase transition in a multiplex network, while (b) adaptive interlayer coupling $\lambda r^{a} r^{b}$ leads to ES. Two different types of transitions ( $r^{a}$ vs $\sigma$ ) are shown in (a) and (b) for a multiplex network of two globally connected layers. Here $N=5000$ and $\lambda=0.5$. Circles and bullets with solid lines correspond, respectively, to forward and backward continuation of $\sigma$, unless stated otherwise, throughout the paper.

the absence of an adaptive interlayer coupling, the usual static $\lambda$ gives rise to a continuous phase transition in layer $a$ [Fig. 1(a)]. However, it unfolds that the presence of adaptive interlayer coupling $\lambda r^{a} r^{b}$ strikingly leads to a discontinuous phase transition (ES) accompanied by hysteresis [Fig. 1(b)].

\section{B. Factors determining hysteresis width}

Figure 2 further elaborates on how the interlayer coupling strength and frequency mismatch between the mirror nodes affects the transition to synchronization or to desynchronization in layer $a$. It turns out that an increase in $\lambda$ increases the hysteresis size [Figs. 2(a)-2(c)] associated with the emergent ES. Similarly, for a given $\lambda$, an increase in frequency mismatch $\Delta \omega$ between mirror nodes also increases
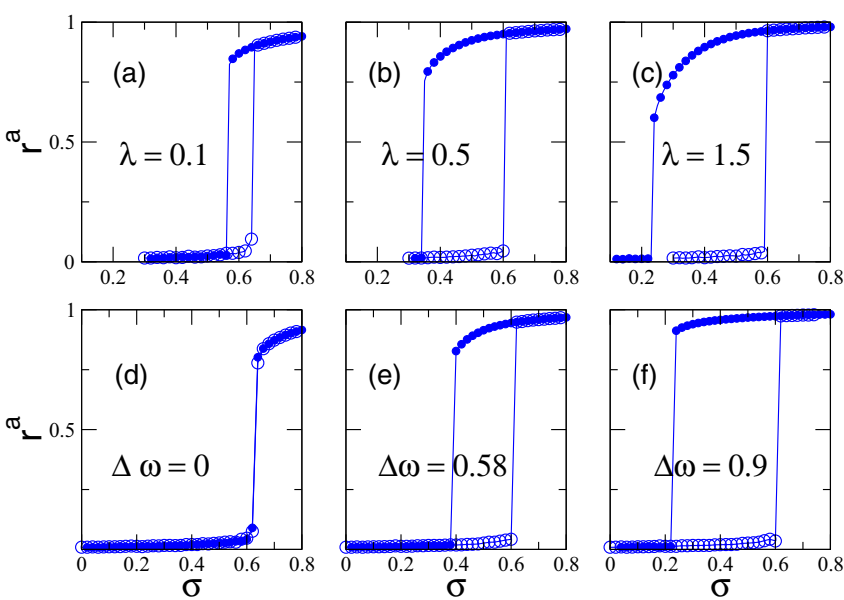

FIG. 2. Plots of (a)-(c) represent $r^{a}$ vs $\sigma$ for different $\lambda$ values with $\Delta \omega$ fixed at 0.58 . Plots of (d)-(f) represent $r^{a}$ vs $\sigma$ for different $\Delta \omega$ values with $\lambda$ fixed at 0.4 . In all the plots $N=10000$. 

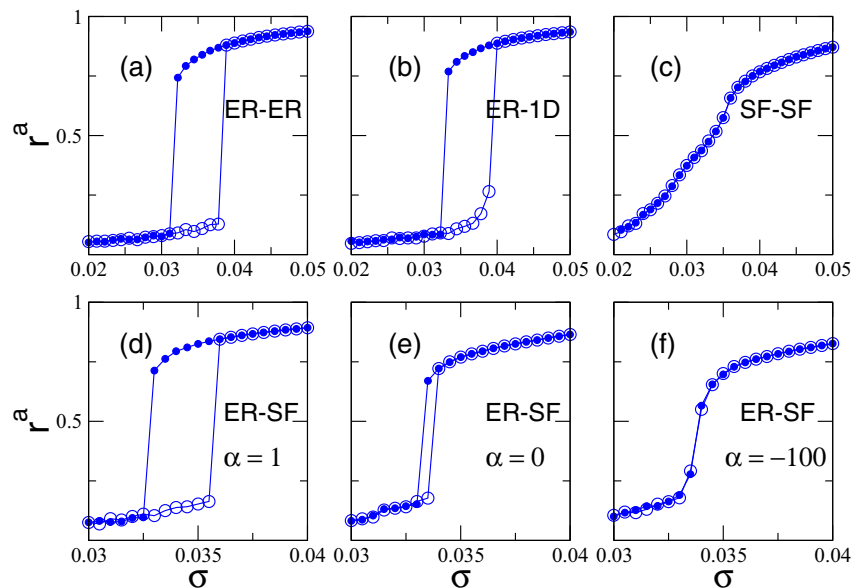

FIG. 3. Plots of $r^{a}$ vs $\sigma$, showing robustness of ES against change in the network topology. Here $N=1000, \lambda=3$, and the average connectivity $\left\langle k^{a(b)}\right\rangle$ of the two layers is $\left\langle k^{a}\right\rangle=\left\langle k^{b}\right\rangle=16$.

the hysteresis size [Figs. 2(d)-2(f)]. To measure the strength of the frequency mismatch between the mirror nodes, we consider

$$
\Delta \omega=1-\frac{1}{2 \sum_{i=1}^{N}\left|\omega_{i}^{a}\right|} \sum_{i=1}^{N}\left|\left(\omega_{i}^{a}+\omega_{i}^{b}\right)\right|,
$$

where $0 \leqslant \Delta \omega \leqslant 1$; in the extreme cases $\Delta \omega=0$ if $\omega_{i}^{a}=\omega_{i}^{b}$ and $\Delta \omega=1$ if $\omega_{i}^{a}=-\omega_{i}^{b}$.

To obtain a desired value of $\Delta \omega$, starting with $\Delta \omega=0$, two pairs of mirror nodes are chosen randomly and their natural frequencies are swapped within the layers. After each swapping, $\Delta \omega$ is recalculated and the change is accepted if the newer value of $\Delta \omega$ is closer to the desired value; otherwise, the change is discarded. This process is repeated until we get a desired value of $\Delta \omega$.

Note that the coupling value at which $r^{a}$ makes a downward jump, $\sigma_{c}^{b}$, manifests a significant decrease with an increase in $\lambda$ as well as $\Delta \omega$, while the changes in the forward critical coupling $\sigma_{c}^{f}$ are very small. Hence, appropriate choices of both $\lambda$ and $\Delta \omega$ determine the threshold of the explosive transition to desynchronization.

\section{Robustness of ES against network topology}

The emergence of ES through interlayer adaptation largely remains robust against the changes in the network topology of the individual layers. However, an increase in the degree heterogeneity of one or both of the layers can cause suppression of ES, eventually leading to a continuous phase transition for a very high degree heterogeneity. To demonstrate the impact of the network topology and the degree heterogeneity, we consider a regular ring network [43], an Erdős-Rényi (ER) random network [44], and scale-free (SF) networks with different values of degree heterogeneity [45] for the multiplexed layers. In the numerical simulations we replace intralayer coupling $\sigma^{a(b)} / N$ in Eq. (1) by $\sigma^{a(b)}$ [46]. Figures 3(a) and 3(b) show that a multiplex network comprised of either ERER or ER-regular-ring networks exhibits ES along with a hysteresis as depicted by the multiplex network comprised of the globally connected layers [Figs. 1(b) and 2]. However, ES disappears for both layers having heterogeneous degree distributions [Fig. 3(c)].

To systematically analyze the effect of the degree heterogeneity on the nature of the transition to synchronization, we fix the layer $a$ to an ER network while the degree heterogeneity of the layer $b$ is varied. Heterogenous networks with $p(k) \propto k^{-\gamma}$ having different $\gamma$ values can be generated by incorporating aging of the nodes [45]. Here $\gamma$ determines the heterogeneity of the degree distribution. In a growing network model, the probability of a newly added node to connect with an existing node $i$ is given by $p_{i} \propto k_{i} \tau_{i}^{-\alpha}$, where $k_{i}$ and $\tau_{i}$ are the degree and the age of the $i$ th node, respectively. The parameter $\alpha=-100,0,1$ corresponds to $\gamma \approx 2,3, \infty$, respectively [45]. Figures 2(d) and 2(e) show that the jump size as well as the hysteresis size decreases with a decrease in the value of $\alpha$ from 1 to 0 . With a further decrease in $\alpha$ to -100 , the hysteresis disappears [Fig. 3(f)].

\section{Mean-field analysis for $\Delta \omega=1$}

For an infinite network size, we analytically derive $r^{a(b)}$ from the synchronized state. The natural frequency of an $i$ th node in layer $a$ is drawn from a uniform distribution, i.e., $g\left(\omega^{a}\right)=1 / 2 \gamma$, where $\gamma=0.5$. Now we take $\Delta \omega=1$, i.e., $\omega_{i}^{b}=-\omega_{i}^{a}$. Equation (1) can be rewritten in terms of the order parameter (2) as

$$
\begin{aligned}
& \dot{\theta_{i}^{a}}=\omega_{i}^{a}+\sigma r^{a} \sin \left(\psi^{a}-\theta_{i}^{a}\right)+\lambda A \sin \left(\theta_{i}^{b}-\theta_{i}^{a}\right), \\
& \dot{\theta_{i}^{b}}=-\omega_{i}^{a}+\sigma r^{b} \sin \left(\psi^{b}-\theta_{i}^{b}\right)+\lambda A \sin \left(\theta_{i}^{a}-\theta_{i}^{b}\right),
\end{aligned}
$$

where $i=1, \ldots, N$ and $A=r^{a} r^{b}$. In the synchronous state $\dot{\theta}_{i}^{a(b)}=\bar{\omega}[15,47]$, where $\bar{\omega}=\frac{1}{2 N} \sum_{i=1}^{N}\left(\omega_{i}^{a}+\omega_{i}^{b}\right)=0$; hence the synchronous state is a fixed point, i.e., $\dot{\theta}_{i}^{a(b)}=0$. If $r^{a}=$ $r^{b}=r$ and $\psi^{a}=-\psi^{b}$, Eqs. (3a) and (3b) suggest that values of the phases corresponding to a fixed point state should be such that $\theta_{i}^{a}=-\theta_{i}^{b}$. By substituting these phase values in Eq. (2), one gets $r^{a}=r^{b}=r$ and $\psi^{a}=-\psi^{b}$ validating the assumption. On assuming $\psi^{a}=\psi^{b}=0$ [47], Eq. (3a) results in

$$
\omega_{i}^{a}-\sigma r \sin \left(\theta_{i}^{a}\right)-\lambda A \sin \left(2 \theta_{i}^{a}\right)=0 .
$$

After substituting $\sin \left(2 \theta_{i}^{a}\right)= \pm 2 \sin \left(\theta_{i}^{a}\right) \sqrt{1-\sin ^{2}\left(\theta_{i}^{a}\right)}$, Eq. (4) results in a fourth-order polynomial $x_{i}^{4}+p_{i} x_{i}^{3}+$ $q_{i} x_{i}^{2}+r_{i}^{\prime} x_{i}+s_{i}=0$, where $x_{i}=\sin \left(\theta_{i}^{a}\right)$ and the coefficients are given as

$$
\begin{aligned}
& p_{i}=0, \quad q_{i}=\frac{\sigma^{2} r^{2}}{4 \lambda^{2} A^{2}}-1, \\
& r_{i}^{\prime}=-\frac{2 \omega_{i}^{a} \sigma r}{4 \lambda^{2} A^{2}}, \quad s_{i}=\frac{\left(\omega_{i}^{a}\right)^{2}}{4 \lambda^{2} A^{2}} .
\end{aligned}
$$

The roots of the polynomial are [48]

$$
\begin{aligned}
& x_{i_{1,2}}=\frac{R_{i} \pm D_{i}}{2}, \\
& x_{i_{3,4}}=-\frac{R_{i} \pm E_{i}}{2},
\end{aligned}
$$



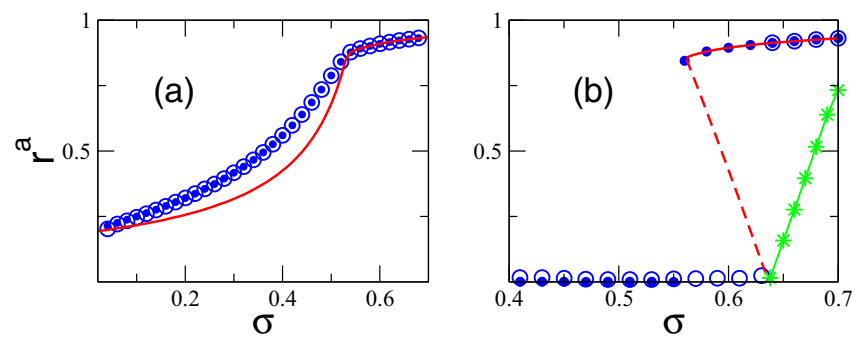

FIG. 4. Plots of $r^{a}$ vs $\sigma$, (a) without adaptive interlayer coupling and (b) with adaptive interlayer coupling. The solid line, dashed line, and solid line with stars represent analytically predicted $r$ values from the solutions of Eq. (11). Here $\omega_{i}^{a}=-0.5+(i-1) /(N-1)$ $(i=1,2, \ldots, N), \Delta \omega=1, \lambda=0.1$, and $N=10000$.

where

$$
\begin{aligned}
& R_{i}=\sqrt{z_{i}-q_{i}}, \\
& D_{i}= \begin{cases}\sqrt{-R_{i}^{2}-2 q_{i}-2 r_{i}^{\prime} / R_{i}} & \text { for } R_{i} \neq 0 \\
\sqrt{-2 q_{i}+2 \sqrt{z_{i}^{2}-4 s_{i}}} & \text { for } R_{i}=0,\end{cases} \\
& E_{i}= \begin{cases}\sqrt{-R_{i}^{2}-2 q_{i}+2 r_{i}^{\prime} / R_{i}} & \text { for } R_{i} \neq 0 \\
\sqrt{-2 q_{i}-2 \sqrt{z_{i}^{2}-4 s_{i}}} & \text { for } R_{i}=0 .\end{cases}
\end{aligned}
$$

Here $z_{i}$ is a real root of the polynomial $z_{i}^{3}-q_{i} z_{i}^{2}-4 s_{i} z_{i}+$ $4 q_{i} s_{i}-r_{i}^{\prime 2}=0$. Out of the four roots, the root corresponding to the physically accepted solution is selected as follows. Equation (4) suggests that for any two nodes $i$ and $j$, if $\omega_{j}^{a}=-\omega_{i}^{a}$, we get $\theta_{j}^{a}=-\theta_{i}^{a}$. Therefore, for the synchronous solution, we consider only that root of the polynomial which satisfy the above condition for the phases. Equation (6) shows that if $\omega_{j}^{a}=-\omega_{i}^{a}, R_{i}=R_{j}$ because $q_{i}$ is independent of $\omega_{i}^{a}$ and the third-order polynomial also does not depend on the sign of $\omega_{i}^{a}$; therefore, $z_{i}$ becomes independent of the sign of $\omega_{i}^{a}$. Moreover, for any nonzero value of $\sigma, r$, and $\omega_{i}^{a}, R_{i} \neq 0$ because if $R_{i}=0$ or $z_{i}=q_{i}$, the third-order polynomial would imply $r_{i}^{\prime}=0$, which is not possible unless $\lambda$ is infinite. Equation (6) also implies that $D_{i}=E_{j}$; therefore $x_{i_{1(3)}}=-x_{j_{3(1)}}$ and $x_{i_{2(4)}}=-x_{j_{4(2)}}$. Thus, a physically accepted root is either from $x_{i_{1}}$ and $x_{i_{3}}$ or from $x_{i_{2}}$ and $x_{i_{4}}$. We find that only the $r$ values corresponding to $x_{i_{2}}\left(\omega_{i}^{a}>0\right)$ and $x_{i_{4}}\left(\omega_{i}^{a}<0\right)$ match with those of numerical simulations [see the solid red line in Fig. 4(b)]; hence the physically accepted root should be $x_{i_{2}}$ for $\omega_{i}^{a}>0$ and $x_{i_{4}}$ for $\omega_{i}^{a}<0$.

In Eq. (2) the summation can be broken into two parts, one arising due to the locked oscillators and the other one due to the drifting oscillators as follows:

$$
r^{a} e^{i \psi^{a}}=r_{l}^{a} e^{i \psi_{l}^{a}}+r_{d}^{a} e^{i \psi_{d}^{a}}
$$

Here the subscripts $l$ and $d$ represent the locked and the drifting oscillators, respectively. For a given $\sigma$ and $\lambda$, the natural frequency of the locked oscillator must satisfy the relation

$$
\left|\omega_{i}^{a}\right| \leqslant \max \left|\sigma r \sin \left(\theta_{i}^{a}\right)+\lambda A \sin \left(2 \theta_{i}^{a}\right)\right| .
$$

For $f\left(\theta_{i}^{a}\right)$ representing the right-hand side (RHS) of Eq. (8), it has extrema at

$$
\cos \left(\theta_{i}^{a}\right)_{ \pm}^{*}=\frac{-\sigma r}{8 \lambda A} \pm \sqrt{\frac{\sigma^{2} r^{2}}{64 \lambda^{2} A^{2}}+0.5},
$$

obtained from the roots of $d f / d \theta_{i}^{a}=0$. Substituting the condition for extrema in Eq. (8), we get

$$
\left|\omega_{i}^{a}\right| \leqslant\left|\sin \left(\theta_{i}^{a}\right)_{ \pm}^{*}\left\{\frac{3 \sigma r \pm \sqrt{\sigma^{2} r^{2}+32 \lambda^{2} A^{2}}}{4}\right\}\right| .
$$

Since $f\left\{\left(\theta_{i}^{a}\right)_{+}^{*}\right\}>f\left\{\left(\theta_{i}^{a}\right)_{-}^{*}\right\}$, only $\cos \left(\theta_{i}^{a}\right)_{+}^{*}$ is considered to determine all the locked oscillators. Equation (9) manifests that an increase in $\lambda$ leads to an increase in the RHS of Eq. (9), indicating that the same number of nodes can get synchronized even at a much smaller $\sigma$ value, in turn causing a decrease in the value of $\sigma_{c}^{b}$ [Figs. 2(a)-2(c)].

In the infinite-size limit, the probability of finding an oscillator having the natural frequency $\omega^{a}$, phase $\theta^{a}$, and its mirror node's phase being $\theta^{b}$ is $\rho^{a}\left(\theta^{a}, \theta^{b}, \omega^{a}\right)=c\left(\omega^{a}\right) /\left|\dot{\theta}^{a}\right|$, where $c\left(\omega^{a}\right)$ is a constant determined from the relation $\int_{-\pi}^{\pi} \int_{-\pi}^{\pi} \rho^{a}\left(\theta^{a}, \theta^{b}, \omega^{a}\right) d \theta^{a} d \theta^{b}=1$ [49]. It follows that the second term on the RHS of Eq. (7) can be written as

$$
\begin{aligned}
r_{d}^{a} e^{i \psi_{d}^{a}}= & \int_{-\pi}^{\pi} \int_{-\pi}^{\pi} \int_{\left|\omega^{a}\right|>\gamma_{c}} d \theta^{a} d \theta^{b} d \omega^{a} \\
& \times \frac{c\left(\omega^{a}\right) e^{i \theta^{a}} g\left(\omega^{a}\right)}{\left|\omega^{a}-\sigma r \sin \left(\theta^{a}\right)+\lambda A \sin \left(\theta^{b}-\theta^{a}\right)\right|} .
\end{aligned}
$$

The $\gamma_{c}$ in Eq. (10) is equal to the RHS of Eq. (9). Additionally, $g\left(-\omega^{a}\right)=g\left(\omega^{a}\right)$ and $\rho^{a}\left(\theta^{a}+\pi, \theta^{b},-\omega^{a}\right)=\rho^{a}\left(\theta^{a}, \theta^{b}, \omega^{a}\right)$. Following the arguments of Ref. [47], we get $r_{d}^{a}=0$.

Taking $\psi^{a}=0$ and $r^{a}=r$, Eq. (7) can be rewritten as

$$
r=\int_{-\gamma_{c}}^{\gamma_{c}} \sqrt{1-\sin ^{2}\left(\theta^{a}\right)} g\left(\omega^{a}\right) d \omega^{a},
$$

where $g\left(\omega^{a}\right)=1$ for $\left|\omega^{a}\right| \leqslant 0.5$ and 0 for $\left|\omega^{a}\right|>0.5$. After plugging the values of phases from Eq. (5) into Eq. (11), we numerically calculate the roots ( $r^{a}$ values) of Eq. (11). Figures 4(a) and 4(b) show that the analytical prediction of $r^{a}$ is in fair agreement with its numerical estimation. In Fig. 4(b) the solid and the dashed lines are two different solutions of Eq. (11) corresponding to the root $x_{i_{2(4)}}$, whereas the line with stars is the solution corresponding to the root $x_{i_{1}}$ for $\omega_{i}^{a}>0$ and $x_{i_{3}}$ for $\omega_{i}^{a}<0$. If there exist two values of $r^{a}$ for a given set of $\sigma$ and $\lambda$, the line with stars shows only the largest of them. It is obvious that only $x_{i_{2(4)}}$ represents the synchronous state. Figure 4(a) shows that in the absence of interlayer adaptation $r^{a}$ increases gradually, yielding a partially synchronized state, or a giant cluster of all the nodes having $\dot{\theta}_{i}^{a}=0$ grows in size by recruiting more and more nodes in it as $\sigma$ increases. The difference between the numerics and the analytical solution at $\sigma \approx 0.4$ is approximately 0.1 , which might be arising due to the drifting oscillators in the numerical simulations. However, for the smaller and the larger values of $\sigma$ the difference is negligible. In the presence of adaptation, Eq. (11) does not have any nonzero solution for $\sigma<\sigma_{c}^{b}$, whereas at $\sigma_{c}^{b}$ one observes an abrupt transition to $r^{a} \approx 0.8$ [Fig. 4(b)]. The solid line in Fig. 4(b) represents the stable synchronized state, and the dashed line joining the stable state to the incoherent state should then represent the unstable state $[14,15,50,51]$. 
The unstable state exists due to the simultaneous presence of two stable states: the incoherent state and the coherent state. Although not shown in Fig. 4(b), $r^{a}=r=0$ is also a solution of Eq. (11) for all the $\sigma$ values. At $r^{a}=0$, the RHS of Eq. (11) is zero; hence $r^{a}=0$ is always a solution of Eq. (11).

\section{E. Adaptive interlayer coupling suppresses the giant cluster}

The introduction of adaptive interlayer coupling suppresses the formation of the giant cluster, which in turn leads to ES. To demonstrate this suppression, we evaluate the RHS of Eq. (9) at $r=0$ for the two cases $A=1$ and $A=r^{2}$. Further, to simplify the calculations we take $\sigma=\lambda$. For $A=1$ and $A=r^{2}=0, \cos \left(\theta_{i}^{a}\right)_{+}^{*} \approx 0.7$ and 0 , respectively, and hence $\sin \left(\theta_{i}^{a}\right)_{+}^{*} \approx 0.7$ and 1 , respectively; thus the contributions from this term for both cases remain almost the same. However, the term in large curly bracket on the RHS for the two cases is $\sqrt{2} \lambda$ and 0 . Therefore, the adaptive coupling leads to suppression of the number of nodes in the partial synchronous state. In other words, the formation of the giant cluster is suppressed for all $\sigma<\sigma_{c}^{f}$.

\section{F. Mean-field analysis for $\Delta \omega \neq 1$}

For globally connected layers, using the mean-field analysis, we prove that the hysteresis size increases with an increase in $\Delta \omega$. To show this we calculate $\sigma_{g c}^{b}$, the minimum $\sigma$ value below which a globally synchronous state cannot exist. Note that, in general, $\sigma_{g c}^{b} \geqslant \sigma_{c}^{b}$, but the transition to desynchronization from a high $r^{a(b)}$ value, unless $\lambda$ is high (Fig. 2), implies that $\sigma_{g c}^{b} \approx \sigma_{c}^{b}$. Using three assumptions $r^{a}=$ $r^{b}=r, \psi^{a}=\psi^{b}=\psi$, and $\cos \left(\psi-\theta_{i}^{a(b)}\right)=\left\{1-\sin ^{2}(\psi-\right.$ $\left.\left.\theta_{i}^{a(b)}\right) / 2\right\}$, the phases in the synchronous state can be written in the form of Eq. (A6). To find $\sigma_{g c}^{b}$, Eq. (2) is numerically solved by substituting $\sin \left(\psi-\theta_{i}^{a}\right)$ values from Eq. (A6). Figure 5 corroborates that an increase in $\Delta \omega$ results in an increase of the hysteresis size $\left(\sigma_{c}^{f}-\sigma_{g c}^{b}\right)$. Note that $\sigma_{c}^{f}$ is independent of $\Delta \omega$. The values obtained here for $\sigma_{c}^{f}$ can also be obtained from Ref. [52] which shows that for $N \rightarrow \infty$ the incoherent state loses its stability at $\sigma_{c}^{f}=4 \gamma / \pi \approx 0.636$.

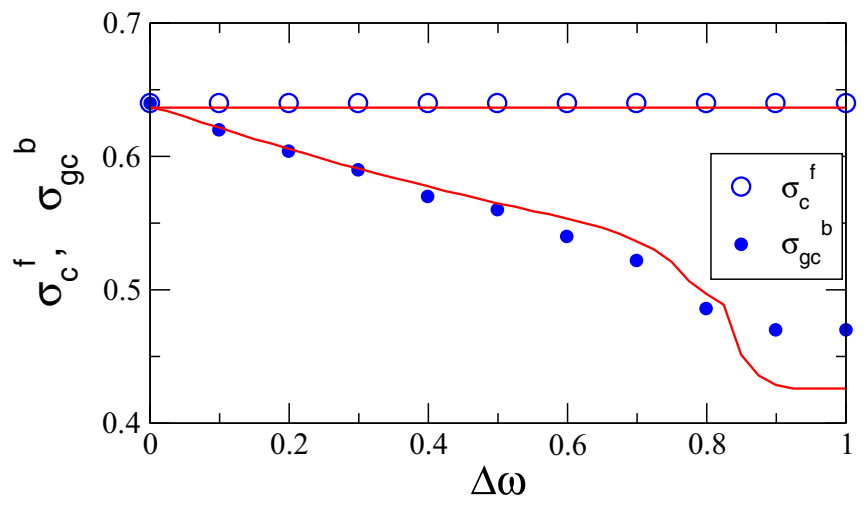

FIG. 5. Plot of $\sigma_{c}^{f}$ and $\sigma_{g c}^{b}$ as a function of $\Delta \omega$. Here $\omega_{i}^{a}=-0.5+(i-1) /(N-1)(i=1,2, \ldots, N), \lambda=0.2$, and $N=$ 10000 . The upper red line represents the value $4 \gamma / \pi$, with $\gamma=0.5$, and the lower red line represents $\sigma_{g c}^{b}$ values from the analytical calculations.

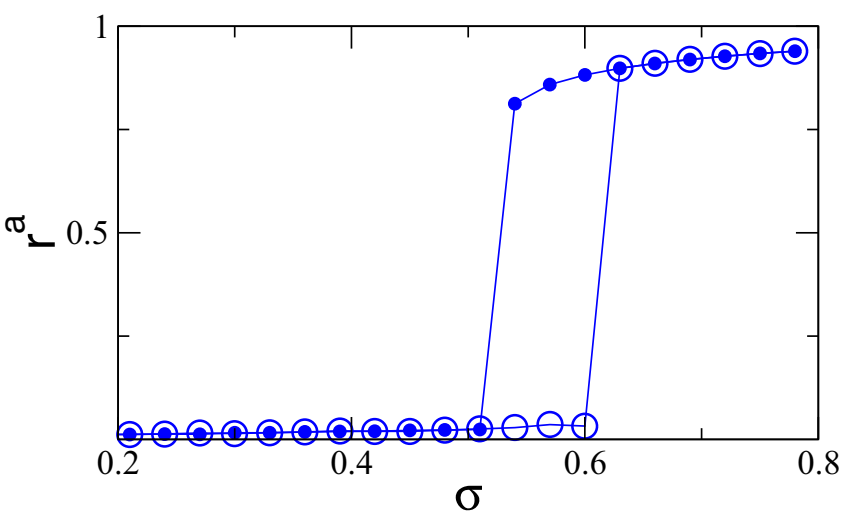

FIG. 6. Plot of $r^{a}$ vs $\sigma$ when adaptive interlayer coupling $\lambda r^{a} r^{b}$ is replaced by $\lambda r^{a}$ in Eq. (1a) and by $\lambda r^{b}$ in Eq. (1b). Here $\Delta \omega=0.33$. $\lambda=0.2$, and $N=10000$.

Further, Fig. 5 shows that the difference between the numerically and analytically found values of $\sigma_{g c}^{b}$ decreases as $\Delta \omega \rightarrow 0$. For $\Delta \omega=0$, phases given by the synchronized state (A6) are exact and therefore the error due to the approximations is zero. We get $\sigma_{g c}^{b}=\sigma_{c}^{f}=4 \gamma / \pi$, as predicted by an isolated network of infinite size (Appendix B). For $\Delta \omega=1$, the difference between numerically and analytically obtained $\sigma_{g c}^{b}$ values is around 0.05 , which can be understood as follows. For $\Delta \omega \rightarrow 1$, Appendix B [Figs. 8(d)-8(g)] shows that analytically calculated $r^{a}$ values are slightly higher than the numerically calculated $r^{a}$ values. Moreover, the natural frequencies of the locked oscillators must satisfy the relation (Appendix B)

$$
\left(\omega_{i}^{a(b)}\right)^{2} \leqslant \frac{4\left(\sigma r^{1 / 3}+2 \lambda r^{4 / 3}\right)^{3}}{27 \lambda} .
$$

The relation above shows that $\sigma_{g c}^{b}$ should be smaller if $r$ is higher, and therefore justifies the observation in Fig. 5.

\section{G. Robustness of ES against change in the adaptive scheme}

Finally, we demonstrate that ES exists even if we replace the interlayer multiplication factor $r^{a} r^{b}$ in Eqs. (1a) and (1b) by $r^{a}$ and $r^{b}$, respectively (Fig. 6). In the infinite-size limit, the addition of a coupling term proportional to $r^{2}$ does not yield any change in the critical coupling at which the incoherent state becomes unstable [52]. Therefore, the $r^{a} r^{b}$ factor in the interlayer coupling only helps us in fixing $\sigma_{c}^{f}$ at a constant value, which otherwise might be sensitive to the parameter $\lambda$ or $\Delta \omega$. Note that in the Zhang et al. model [15], the intralayer coupling term containing $r^{2}$ was shown to be responsible for the occurrence of ES; however, such a condition is not required for the case of adaptive interlayer coupling proposed in this article.

\section{CONCLUSION}

It is known that in a system of networked oscillators any microscopic strategy which can suppress the formation of the giant cluster can eventually lead to ES. It was reported earlier that intralayer adaptive coupling through the local order parameters can trigger ES in a multilayer network with dependent links [15]. The present work showed that an adaptive interlinked setup between the pairs of the mirror 
(interconnected) nodes by means of the global order parameter can also trigger ES in the multiplexed layers. We have discussed in detail how the interlayer coupling strength and the interlayer pairwise frequency correlation enable us to shape the hysteresis of the emergent ES. The occurrence of ES through such a scheme is largely robust against changes in the topology of the individual layers, except for a very high degree heterogeneity of either of the layers. In heterogeneous networks, a partially synchronous state exists at all coupling values [46]. The adaptive interlayer coupling scheme might not be able to suppress the formation of a giant cluster and hence it could not instigate ES in these networks. We provided a mean-field analytical treatment to ground the perceived outcomes and substantiate that the analytical predictions are in fair agreement with the numerical estimations.

Our model has some level of similarity to several realworld complex systems having a multilayer underlying network structure. For example, large-scale brain multilayer networks can be defined based on the functional interdependence of the brain regions [53]. We expect that our investigation of ES originating from the interlayer adaptation would forward the understanding of discontinuous phase transitions existing in intertwined real-world complex dynamical systems.

\section{ACKNOWLEDGMENTS}

S.J. and A.D.K. acknowledge the Government of India for financial support through CSIR Grant No. 25(0293)/18/EMRII. S.J. also acknowledges DST Grant No. EMR/2016/001921. A.K. acknowledges CSIR (Government of India) for financial support through SRF.

\section{APPENDIX A: GLOBALLY SYNCHRONIZED STATE FOR $\Delta \omega \neq 1$}

For the general case of $\Delta \omega \neq 1$ we derive phases in the globally synchronized state. For the globally connected layers, rewriting Eq. (1) in terms of the order parameter (2),

$$
\begin{aligned}
& \dot{\theta_{i}^{a}}=\omega_{i}^{a}+\sigma r^{a} \sin \left(\psi^{a}-\theta_{i}^{a}\right)+\lambda r^{a} r^{b} \sin \left(\theta_{i}^{b}-\theta_{i}^{a}\right), \\
& \dot{\theta}_{i}^{b}=\omega_{i}^{b}+\sigma r^{b} \sin \left(\psi^{b}-\theta_{i}^{b}\right)+\lambda r^{a} r^{b} \sin \left(\theta_{i}^{a}-\theta_{i}^{b}\right),
\end{aligned}
$$

where $i=1, \ldots, N$. In the globally synchronous state we have $\dot{\theta}_{i}^{a(b)}=\bar{\omega} \forall i$ [15], where $\bar{\omega}$ is the mean of the natural frequencies of the entire multiplex network. Furthermore, we take $\bar{\omega}=0$ and hence the synchronous state is a fixed point solution. On assuming $\psi^{a} \approx \psi^{b}=\psi$ and $r^{a} \approx r^{b}=r$, we add Eqs. (A1a) and (A1b), yielding

$$
\sigma r \sin \left(\psi-\theta_{i}^{b}\right)=-\omega_{i}^{a}-\omega_{i}^{b}-\sigma r \sin \left(\psi-\theta_{i}^{a}\right) .
$$

We substitute Eq. (A2) in Eq. (A1b) with the interlayer coupling written as $\sin \left(\theta_{i}^{a}-\theta_{i}^{b}\right)=\sin \left(\theta_{i}^{a}-\psi\right) \cos \left(\psi-\theta_{i}^{b}\right)+$ $\cos \left(\theta_{i}^{a}-\psi\right) \sin \left(\psi-\theta_{i}^{b}\right)$, where $\cos \left(\psi-\theta_{i}^{a(b)}\right) \approx \pm\{1-$ $\left.\sin ^{2}\left(\psi-\theta_{i}^{a(b)}\right) / 2\right\}$. Note that the higher-order terms in $\cos \left(\psi-\theta_{i}^{a(b)}\right)$ can be neglected as $r^{a(b)} \approx 1$ for the synchronous state implies that $\theta_{i}^{a(b)} \approx \psi$ [Eq. (2)]. Moreover, it also indicates that only the positive value of $\cos \left(\psi-\theta_{i}^{a(b)}\right)$ must be considered. After some mathematical simplifications, Eq. (A1b) results in a third-order polynomial $x_{i}^{3}+p_{i} x_{i}^{2}+$ $q_{i} x_{i}+r_{i}^{\prime}=0$, where $x_{i}=\sin \left(\psi-\theta_{i}^{a}\right)$. The coefficients of the polynomial are

$$
\begin{aligned}
p_{i} & =\frac{\left(\omega_{i}^{a}+\omega_{i}^{b}\right) 3 \lambda r}{2 \sigma r^{2} \lambda}, \\
q_{i} & =\frac{1}{\lambda r^{2}}\left\{-\sigma r-2 \lambda r^{2}+\frac{\left(\omega_{i}^{a}+\omega_{i}^{b}\right)^{2} \lambda}{\left(2 \sigma^{2}\right)}\right\}, \\
r_{i}^{\prime} & =-\frac{1}{\lambda r^{2}}\left\{\omega_{i}^{a}+\frac{\left(\omega_{i}^{a}+\omega_{i}^{b}\right) \lambda r}{\sigma}\right\} .
\end{aligned}
$$

The roots of the polynomial are given by [48]

$$
\begin{gathered}
x_{i_{1}}=U_{i}+V_{i}-\frac{p_{i}}{3}, \\
x_{i_{2}}=-\frac{U_{i}+V_{i}}{2}+i \frac{\sqrt{3}}{2} \frac{U_{i}-V_{i}}{2}-\frac{p_{i}}{3}, \\
x_{i_{3}}=-\frac{U_{i}+V_{i}}{2}-i \frac{\sqrt{3}}{2} \frac{U_{i}-V_{i}}{2}-\frac{p_{i}}{3},
\end{gathered}
$$

where

$$
\begin{aligned}
& U_{i}=\left\{\frac{-b_{i}}{2}+\sqrt{\frac{b_{i}^{2}}{4}+\frac{a_{i}^{3}}{27}}\right\}^{1 / 3}, \\
& V_{i}=\left\{\frac{-b_{i}}{2}-\sqrt{\frac{b_{i}^{2}}{4}+\frac{a_{i}^{3}}{27}}\right\}^{1 / 3},
\end{aligned}
$$

with $a_{i}=\left(3 q_{i}-p_{i}^{2}\right) / 3$ and $b_{i}=\left(2 p_{i}^{3}-9 p_{i} q_{i}+27 r_{i}^{\prime}\right) / 27$.

A physically acceptable root should be such that for $\sigma \rightarrow \infty, x_{i} \rightarrow 0$. Moreover, as $\sigma \rightarrow \infty, p_{i} \rightarrow 0, q_{i} \rightarrow-\infty$, and $r_{i}^{\prime} \rightarrow-\omega_{i}^{a} / \lambda r^{2}$. Therefore, $a_{i} \rightarrow-\infty$ and $b_{i} \rightarrow\left(\omega_{i}^{b}-\right.$ $\left.\omega_{i}^{a}\right) / 2 \lambda r^{2}$; in turn, $\frac{b_{i}^{2}}{4}+\frac{a_{i}^{3}}{27} \rightarrow-\infty$. A negative value of $\frac{b_{i}^{2}}{4}+$ $\frac{a_{i}^{3}}{27}$ implies that $U_{i}$ and $V_{i}$ are complex numbers [48]. Writing the roots in another form leads to

$$
x_{i_{k}}=2 \sqrt{\frac{-a_{i}}{3}} \cos \left(\frac{\phi_{i}}{3}+\frac{2(k-1) \pi}{3}\right)-\frac{p_{i}}{3},
$$

where $k=1,2,3$ and $\phi_{i}=\cos ^{-1}\left( \pm \sqrt{\frac{b_{i}^{2} / 4}{-a_{i}^{3} / 27}}\right)$ for $b_{i} \lessgtr 0$. For $\sigma \rightarrow \infty, \phi_{i} \rightarrow \pi / 2$ and therefore at infinite coupling we get $x_{i_{1}}=2 \sqrt{\frac{-a_{i}}{3}} \cos \left(\frac{\pi}{6}\right), x_{i_{2}}=2 \sqrt{\frac{-a_{i}}{3}} \cos \left(\frac{5 \pi}{6}\right)$, and $x_{i_{3}}=$ $2 \sqrt{\frac{-a_{i}}{3}} \cos \left(\frac{3 \pi}{2}\right)$. The first two roots $x_{i_{1}}$ and $x_{i_{2}}$ diverge while $x_{i_{3}} \rightarrow 0$. Note that in $x_{i_{3}}, \sqrt{\frac{-a_{i}}{3}}$ increases while $\cos \left(\frac{\phi_{i}}{3}+\frac{4 \pi}{3}\right)$ decreases, and since the decrement is faster [as $\cos \left(\phi_{i}\right)$ is proportional to $\left.a_{i}^{-3 / 2}\right]$ than the increment, the overall product experiences a decrement. Therefore, the physically accepted root is $x_{i_{3}}$.

\section{APPENDIX B: VALIDATION OF THE ASSUMPTIONS}

We prove that the assumptions (a) $r^{a}=r^{b}=r$, (b) $\psi^{a}=$ $\psi^{b}=\psi$, and (c) $\cos \left(\psi-\theta_{i}^{a(b)}\right)=1-\sin ^{2}\left(\psi-\theta_{i}^{a(b)}\right) / 2$ are valid in the numerical and analytical calculations. For the two extreme values of $\Delta \omega$, i.e., for $\Delta \omega=0,1$, it can be easily checked that the synchronized state is consistent with assumptions (a) and (b). At $\Delta \omega=0$, i.e., for $\omega_{i}^{a}=\omega_{i}^{b}=$ $\omega_{i}$, we get $a_{i}<0$ and $b_{i}=0$. Therefore, $b_{i}^{2} / 4+a_{i}^{3} / 27$ is 

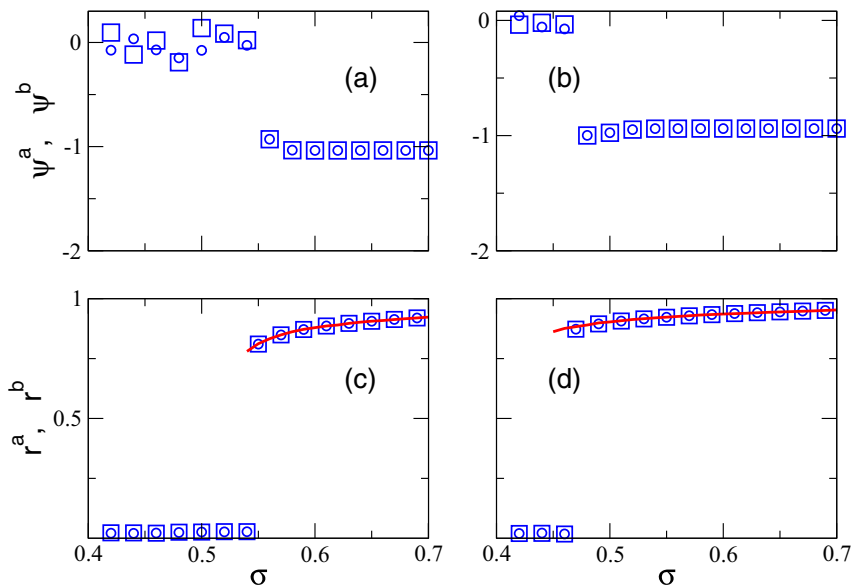

FIG. 7. Plots of (a) and (b) shows time-averaged $\psi^{a}$ (circles) and $\psi^{b}$ (squares) in the backward continuation of $\sigma$. Plots of (c) and (d) shows $r^{a}$ (circles) and $r^{b}$ (squares) in the backward continuation of $\sigma$. In (a) and (c) $\Delta \omega=0.381$, whereas in (b) and (d) $\Delta \omega=0.781$. All the plots have $\lambda=0.2$ and $N=10000$. The solid red line in (c) and (d) represents analytically predicted $r^{a}$ values.

negative and the roots can be represented in the form given by Eq. (A8). The first term in Eq. (A8) is zero because $\phi_{i}=\pi / 2$, and therefore $\sin \left(\psi-\theta_{i}^{a}\right)=-p_{i} / 3=-\omega_{i} / \sigma r$. Putting this in Eq. (A2), we get $\sin \left(\psi-\theta_{i}^{b}\right)=-\omega_{i} / \sigma r$. Therefore, $\theta_{i}^{a}=$ $\theta_{i}^{b}$, and for an identical distribution of phases (2) returns $r^{a}=$ $r^{b}=r$ and $\psi^{a}=\psi^{b}=\psi$. At $\theta_{i}^{a}=\theta_{i}^{b}$, the interlayer coupling term in Eq. (A1) gets canceled and therefore the phase values in this case are exact. Notice that the synchronized states for a multiplex network and for an isolated network are the same [54].

To prove that assumptions (a) and (b) are valid for $\Delta \omega=$ 1 , we proceed as follows. The quantity $\frac{b_{i}^{2}}{4}+\frac{a_{i}^{3}}{27}$ cannot be positive, because it would imply that

$$
\left(\omega_{i}^{a(b)}\right)^{2}>\frac{4\left(\sigma r^{1 / 3}+2 \lambda r^{4 / 3}\right)^{3}}{27 \lambda} .
$$

The relation above shows that oscillators with natural frequencies farther from $\bar{\omega}=0$ are locked, while the oscillators closer to $\bar{\omega}=0$ are not locked, which is contrary to Eq. (9). Therefore, $\frac{b_{i}^{2}}{4}+\frac{a_{i}^{3}}{27} \leqslant 0$, and the phases can be written in the form of Eq. (A8). For the considered natural frequency
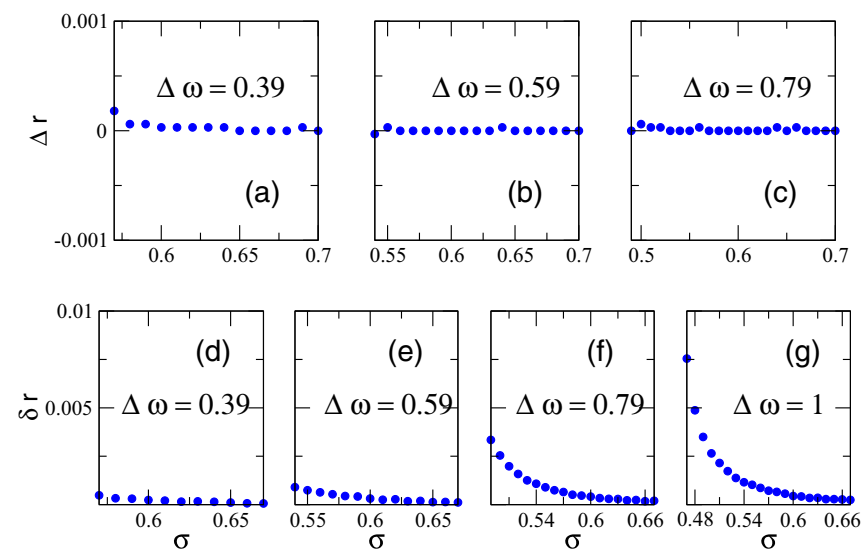

FIG. 8. Plots of (a)-(c) show $\Delta r$ vs $\sigma$ while (d)-(g) show $\delta r$ vs $\sigma . \Delta r$ represents the difference between analytically calculated $r$ values for layer $a$ and layer $b$. $\delta r$ represents the difference between analytically and numerically calculated $r$ values for layer $a$. The network parameters are same as in Fig. 5.

distribution in Fig. $5 \omega_{i}^{a}=-\omega_{N-i+1}^{a}$, where $i=1,2, \ldots$, $N / 2$. From Eq. (A8) it can be checked that $\sin \left(\psi-\theta_{i}^{a}\right)=$ $-\sin \left(\psi-\theta_{N-i+1}^{a}\right)$. Equation (A2) gives $\sin \left(\psi-\theta_{i}^{a}\right)=$ $-\sin \left(\psi-\theta_{i}^{b}\right)$, and therefore $\theta_{i}^{b}=\theta_{N-i+1}^{a}$. Similarly, it can be checked that $\theta_{N-i+1}^{b}=\theta_{i}^{a}$. The distribution of the phases is identical in the two layers; hence Eq. (2) returns $r^{a}=r^{b}=r$ and $\psi^{a}=\psi^{b}=\psi$.

For intermediate $\Delta \omega$ values, Fig. 7 reveals that numerical calculations agree with assumptions (a) and (b). Moreover, analytically and numerically calculated $r^{a}$ values are also in fair agreement [Figs. 7(c) and 7(d)]. Analytical evaluation of $r^{a}$ and $r^{b}$ further elaborates on the validity of assumption (a) [Figs. 8(a)-8(c)]. Next we prove the validity of assumption (c). Note that $\cos \left(\psi-\theta_{i}^{a(b)}\right) \leqslant 1-\sin ^{2}\left(\psi-\theta_{i}^{a(b)}\right) / 2$; here equality holds only for $\theta_{i}^{a(b)}=\psi$. Therefore, assumption (c) shifts the phases closer to $\psi$, and hence we should get a higher $r^{a}$ value from the analytical calculations. Figures $8(\mathrm{~d})-8(\mathrm{~g})$ reveal that the difference between analytically and numerically found $r^{a}$ values, $\delta r$, is marginally positive. Further, $\delta r$ grows as $\Delta \omega \rightarrow 1$; at $\Delta \omega=1$ we get maximum $\delta r \approx 0.01$. An increment in the $\sigma$ value leads to a decrement in the $\delta r$ value. As expected, it is due to the fact that $\theta_{i}^{a(b)} \rightarrow \psi$ as $\sigma \rightarrow \infty$
[1] S. Boccaletti, J. Almendral, S. Guan, I. Leyva, Z. Liu, I. Sendiña-Nadal, Z. Wang, and Y. Zou, Phys. Rep. 660, 1 (2016).

[2] R. M. D’Souza, J. Gómez-Gardeñes, J. Nagler, and A. Arenas, Adv. Phys. 68, 123 (2019).

[3] S. V. Buldyrev, R. Parshani, G. Paul, H. E. Stanley, and S. Havlin, Nature (London) 464, 1025 (2010).

[4] B. A. Huberman and R. M. Lukose, Science 277, 535 (1997).

[5] B. M. Adhikari, C. M. Epstein, and M. Dhamala, Phys. Rev. E 88, 030701(R) (2013).

[6] U. Lee, M. Kim, K. Lee, C. M. Kaplan, D. J. Clauw, S. Kim, G. A. Mashour, and R. E. Harris, Nat. Sci. Rep. 8, 243 (2018).
[7] M. Kim, G. A. Mashour, S.-B. Moraes, G. Vanini, V. Tarnal, E. Janke, A. G. Hudetz, and U. Lee, Front. Comput. Neurosci. 10, 1 (2016).

[8] M. Kim, S. Kim, G. A. Mashour, and U. Lee, Front. Comput. Neurosci. 11, 55 (2017).

[9] J. R. Pomerening, E. D. Sontag, and J. E. Ferell, Jr., Nat. Cell Biol. 5, 346 (2003).

[10] I. Leyva, R. Sevilla-Escoboza, J. M. Buldú, I. Sendiña-Nadal, J. Gómez-Gardeñes, A. Arenas, Y. Moreno, S. Gómez, R. JaimesReátegui, and S. Boccaletti, Phys. Rev. Lett. 108, 168702 (2012). 
[11] P. Kumar, D. K. Verma, P. Parmananda, and S. Boccaletti, Phys. Rev. E 91, 062909 (2015).

[12] H.-A. Tanaka, A. J. Lichtenberg, and S. Oishi, Phys. Rev. Lett. 78, 2104 (1997).

[13] J. Gómez-Gardeñes, S. Gómez, A. Arenas, and Y. Moreno, Phys. Rev. Lett. 106, 128701 (2011).

[14] X. Zhang, X. Hu, J. Kurths, and Z. Liu, Phys. Rev. E 88, 010802(R) (2013).

[15] X. Zhang, S. Boccaletti, S. Guan, and Z. Liu, Phys. Rev. Lett. 114, 038701 (2015).

[16] M. M. Danziger, O. I. Moskalenko, S. A. Kurkin, X. Zhang, S. Havlin, and S. Boccaletti, Chaos 26, 065307 (2016).

[17] C. Zhou and J. Kurths, Phys. Rev. Lett. 96, 164102 (2006).

[18] R. Gutiérrez, A. Amann, S. Assenza, J. Gómez-Gardeñes, V. Latora, and S. Boccaletti, Phys. Rev. Lett. 107, 234103 (2011).

[19] M. De Domenico, A. Solé-Ribalta, E. Cozzo, M. Kivelä, Y. Moreno, M. A. Porter, S. Gómez, and A. Arenas, Phys. Rev. X 3, 041022 (2013).

[20] F. Sorrentino, New J. Phys. 14, 33035 (2012).

[21] S. Boccaletti, G. Bianconi, R. Criado, C. del Genio, J. GómezGardeñes, M. Romance, I. Sendiña-Nadal, Z. Wang, and M. Zanin, Phys. Rep. 544, 1 (2014).

[22] R. Sevilla-Escoboza, I. Sendiña-Nadal, I. Leyva, R. Gutiérrez, J. M. Buldú, and S. Boccaletti, Chaos 26, 065304 (2016).

[23] C. I. del Genio, J. Gómez-Gardeñes, I. Bonamassa, and S. Boccaletti, Sci. Adv. 2, e1601679 (2016).

[24] C. Sarkar, A. Yadav, and S. Jalan, Europhys. Lett. 113, 18007 (2016).

[25] A. Kumar, M. S. Baptista, A. Zaikin, and S. Jalan, Phys. Rev. E 96, 062301 (2017).

[26] E. Pitsik, V. Makarov, D. Kirsanov, N. S. Frolov, M. Goremyko, X. Li, Z. Wang, A. Hramov, and S. Boccaletti, New J. Phys. 20, 075004 (2018).

[27] I. Leyva, I. Sendiña-Nadal, R. Sevilla-Escoboza, V. Vera-Avila, P. Chholak, and S. Boccaletti, Sci. Rep. 8, 8629 (2018).

[28] P. Shinde and S. Jalan, Europhys. Lett. 112, 58001 (2015).

[29] M. Kivelä, A. Arenas, M. Barthelemy, J. P. Gleeson, Y. Moreno, and M. A. Porter, J. Complex Netw. 2, 203 (2014).

[30] S. Osat, A. Faqeeh, and F. Radicchi, Nat. Commun. 8, 1540 (2017).

[31] F. D. Sahneh, C. Scoglio, and P. Van Mieghem, IEEE/ACM Trans. Netw. 21, 1609 (2013).
[32] M. De Domenico, C. Granell, M. A. Porter, and A. Arenas, Nat. Phys. 12, 901 (2018).

[33] M. Diakonova, V. Nicosia, V. Latora, and M. S. Miguel, New J. Phys. 18, 023010 (2016).

[34] S. Jalan and A. Singh, Europhys. Lett. 113, 30002 (2016).

[35] V. Nicosia, P. S. Skardal, A. Arenas, and V. Latora, Phys. Rev. Lett. 118, 138302 (2017)

[36] A. D. Kachhvah and S. Jalan, Europhys. Lett. 119, 60005 (2017).

[37] A. D. Kachhvah and S. Jalan, New J. Phys. 21, 015006 (2019).

[38] S. Jalan, V. Rathore, A. D. Kachhvah, and A. Yadav, Phys. Rev. E 99, 062305 (2019).

[39] S. Jalan, A. Kumar, and I. Leyva, Chaos 29, 041102 (2019).

[40] P. Khanra, P. Kundu, C. Hens, and P. Pal, Phys. Rev. E 98, 052315 (2018).

[41] M. M. Danziger, I. Bonamassa, S. Boccaletti, and S. Havlin, Nat. Phys. 15, 178 (2019).

[42] Y. Kuramoto, in International Symposium on Mathematical Problems in Theoretical Physics, Kyoto, 1975, edited by H. Araki, Lecture Notes in Physics Vol. 39 (Springer, Berlin, 1975).

[43] A.-L. Barabási and R. Albert, Science 286, 509 (1999).

[44] P. Erdös and A. Rényi, Publ. Math. Debrecen 6, 290 (1959).

[45] S. N. Dorogovtsev and J. F. F. Mendes, Phys. Rev. E 62, 1842 (2000).

[46] A. Arenas, A. Díaz Guilera, J. Kurths, Y. Moreno, and C. Zhou, Phys. Rep. 469, 93 (2008).

[47] S. H. Strogatz, Physica D 143, 1 (2000).

[48] Y.-B. Jia, Roots of Polynomials, 2018 (unpublished), available at https://pdfs.semanticscholar.org/2b69/8d3602fe6ce 5ec2b29f70614aa15e36fd397.pdf.

[49] A. Pikovsky, M. Rosenblum, and J. Kurths, Synchronization: A Universal Concept in Nonlinear Sciences (Cambridge University Press, Cambridge, UK, 2003).

[50] V. Vlasov, Y. Zou, and T. Pereira, Phys. Rev. E 92, 012904 (2015).

[51] I. Leyva, I. Sendiña-Nadal, J. A. Almendral, A. Navas, S. Olmi, and S. Boccaletti, Phys. Rev. E 88, 042808 (2013).

[52] S. H. Strogatz and R. E. Mirollo, J. Stat. Phys. 63, 613 (1991).

[53] M. De Domenico, S. Sasai, and A. Arenas, Front. Neurosci. 10, 326 (2016).

[54] D. Pazo, Phys. Rev. E 72, 046211 (2005). 